Check for updates

Cite this: Chem. Sci., 2017, 8, 7983

\title{
Efficient and stable single-doped white OLEDs using a palladium-based phosphorescent excimer $†$
}

\begin{abstract}
Tyler Fleetham, \$ Yunlong Ji, \$ Liang Huang, Trenten S. Fleetham and Jian Li (D) *
A tetradentate $\mathrm{Pd}(\Perp)$ complex, $\mathrm{Pd} 3 \mathrm{O} 3$, which exhibits highly efficient excimer emission is synthesized and characterized. $\mathrm{Pd} 3 \mathrm{O} 3$ can achieve blue emission despite using phenyl-pyridine emissive ligands which have been a mainstay of stable green and red phosphorescent emitter designs, making Pd3O3 a good candidate for stable blue or white OLEDs. Pd3O3 exhibits strong and efficient phosphorescent excimer emission expanding the excimer based white OLEDs beyond the sole class of Pt complexes. Devices of $\mathrm{Pd} 3 \mathrm{O} 3$ demonstrate peak external quantum efficiencies as high as $24.2 \%$ and power efficiencies of $67.9 \mathrm{Lm}$ per $\mathrm{W}$ for warm white devices. Furthermore, Pd3O3 devices in a carefully designed stable structure achieved a device operational lifetime of nearly $3000 \mathrm{~h}$ at $1000 \mathrm{~cd} \mathrm{~m} \mathrm{~m}^{-2}$ without any outcoupling enhancement while simultaneously achieving peak external quantum efficiencies of $27.3 \%$ and power efficiencies over $81 \mathrm{Lm}$ per W.
\end{abstract}

Received 5th June 2017

Accepted 9th September 2017

DOI: $10.1039 / \mathrm{c} 7 \mathrm{sc} 02512 \mathrm{~b}$

rsc.li/chemical-science

white light. ${ }^{16-18}$ However, excimer-based devices face the same operational lifetime challenges as the conventional white OLEDs due to the lack of stable and efficient blue phosphorescent emitters. While many efficient blue emitters have been developed, very few have shown promising operational stabilities. ${ }^{19-26}$ The origin of the low operational stability of existing blue phosphorescent materials is unresolved and is under intensive investigation. ${ }^{27-29}$ The choice of cyclometalating ligands may be one of the major factors leading to the poor operational stability. Many of the most commonly used efficient blue emitters typically contain fluorinated cyclometalating ligands which have been shown to undergo fluorine loss. ${ }^{30,31}$ Furthermore, a recent report demonstrated that blue emitters containing phenyl-azole ligands exhibited much shorter device operational lifetimes than emitters of the same emission energy and similar energy levels which do not contain any fivemembered heterocycles. ${ }^{32}$ Thus, it would be desirable to design blue phosphorescent emitters that employ the cyclometalating ligands similar to those of reported stable green and red phosphorescent emitters (i.e. the absence of fluorine groups or 5-membered heterocycles).

Stable and efficient single-doped WOLEDs require the development of phosphorescent materials with sufficient blue emission, efficient excimer emission, and a molecular design aligned with known stable phosphorescent emitters. However, Ir and Pt complexes have been unable to satisfy this desirable combination of characteristics, necessitating a new avenue of materials development. Pd(II) complexes also have the potential for efficient excimer based white emission due to their square planar molecular geometry. ${ }^{33-35}$ However, Pd(II) complexes have received significantly less attention than their Ir and Pt analogs. This is partially because Pd complexes have typically been

Materials Science and Engineering, Arizona State University, Tempe, AZ, 85287, USA. E-mail: jian.li.1@asu.edu

$\dagger$ Electronic supplementary information (ESI) available. See DOI: $10.1039 / \mathrm{c} 7 \mathrm{sc} 02512 \mathrm{~b}$

\$ These authors contributed equally to this work. 

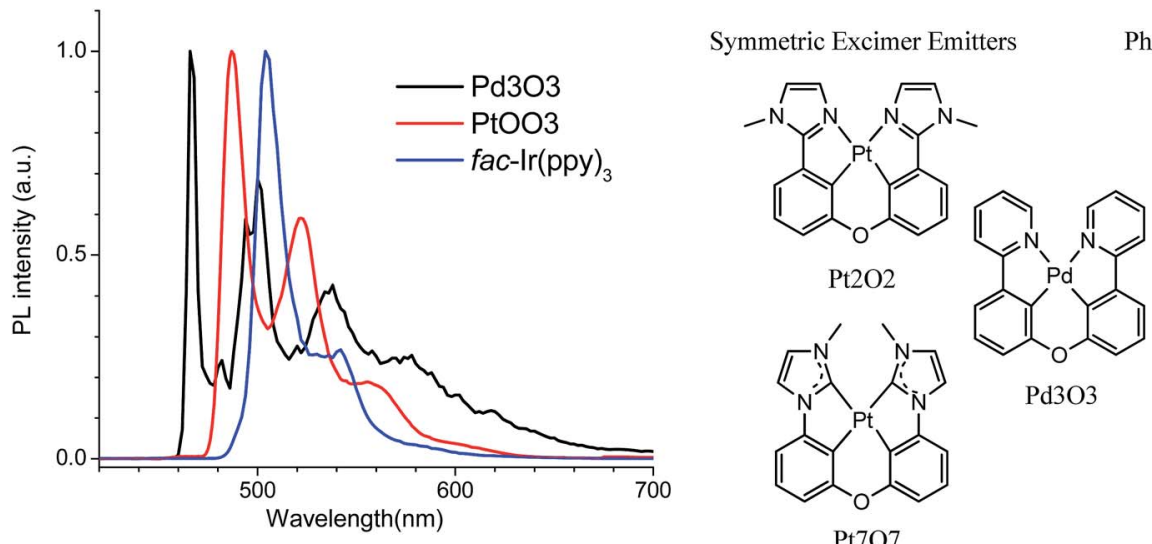

Phenyl-Pyridine Emitters

Fig. 1 Photoluminescent spectra of Pd3O3, PtOO3, and fac- Ir(ppy) 3 at $77 \mathrm{~K}$ in 2-methyl-THF and molecular structures of select tetradentate excimer emitters and phenyl-pyridine based emitters.

non-emissive or weakly emissive due to their low radiative decay rates and low lying metal-centered (MC) states providing nonradiative decay pathways. ${ }^{36,37}$ Furthermore, none of the previous reports of Pd emitters, to our knowledge, have demonstrated efficient excimer emission. In this report, we develop an efficient excimer emitting Pd(II) complex, i.e. Pd3O3 (Fig. 1b), which utilizes a rigid and planar molecular design to achieve efficient blue and white emission while remaining aligned with stable molecular designs. This complex demonstrated efficiencies comparable to its Pt analogs but with higher emission energy and high operational stability. Efficiencies as high as $27.3 \%$ were achieved for $\mathrm{Pd} 3 \mathrm{O} 3$ devices and one device achieved a device operational lifetime of nearly $3000 \mathrm{~h}$ at $1000 \mathrm{~cd} \mathrm{~m}^{-2}$ with an EQE of $19.2 \%$ at that same brightness. This performance establishes Pd complexes as an emerging class of emissive materials and demonstrates their potential for stable, efficient, and simplified white OLEDs.

\section{Design of excimer emitters}

An efficient and stable Pd complex for single doped white OLEDs requires a rigid molecular structure to suppress the nonradiative pathways that afflict many Pd complexes, a planar molecular geometry to allow sufficient excimer emission, and cyclometalating ligands that are aligned with stable emitter designs. The design of symmetric Pt complexes, such as $\mathrm{Pt7O} 7$ and $\mathrm{Pt} 2 \mathrm{O} 2$, offer both rigidity and planar geometry necessary for efficient white emission. ${ }^{38,39}$ However, these complexes have short operational lifetimes possibly resulting from their phenylazole cyclometalating ligands. The designed palladium emitter, $\mathrm{Pd} 3 \mathrm{O} 3$, also has a relatively flat planar geometry as shown in the DFT optimized ground state geometry (Fig. S1 $\dagger$ ) which will make it well suited for excimer emission. Fortunately, Pd3O3, has a higher emission energy than its Pt analogs, allowing high enough triplet energy for warm white emission while using phenyl-pyridine ligands. The comparison of the low temperature emission spectra of $\mathrm{Pd} 3 \mathrm{O} 3$ with its $\mathrm{Pt}$ and Ir analogs are presented in Fig. $1{ }^{40}$ Although the three metal complexes employ the same cyclometalating ligand of phenyl pyridine, the incorporation of palladium metal ions has shifted the maximum emission wavelength of metal complexes from $504 \mathrm{~nm}$ for $f a c-\operatorname{Ir}(\mathrm{ppy})_{3}$ to $466 \mathrm{~nm}$ for Pd3O3, making Pd3O3 suitable as a phosphorescent emitter for blue and white OLED applications. Moreover, the luminescent lifetime of $\mathrm{Pd} 3 \mathrm{O} 3$ at $77 \mathrm{~K}$ is around $165 \mu \mathrm{s}$, much longer than those of their Ir and Pt analogs in the range of microseconds, ${ }^{40,41}$ indicating more localized ligand-centered excited state properties for such Pd complexes.

\section{Photophysical and electrochemical properties}

Both a dilute solution of $\mathrm{Pd} 3 \mathrm{O} 3$ in dichloromethane (DCM) and a dilute thin film ( $1 \%$ by weight $)$ in 2,6-bis $(N$-carbazolyl)pyridine (26mCPy) were prepared for spectral analysis. The absorption of the solution is shown in Fig. 2. The strong absorption peaks below $\sim 360 \mathrm{~nm}$ are assigned to ${ }^{1} \pi-\pi^{*}$ transitions, localized on the phenyl-pyridine ligands. The small shoulder in the $360-450 \mathrm{~nm}$ range is assigned to singlet metal to ligand charge transfer ( $\left.{ }^{1} \mathrm{MLCT}\right)$ transitions similar to previously reported phenyl-pyridine based metal complexes. ${ }^{40,41}$ Both the thin film and solution sample show molecular emission peaks in 460-510 $\mathrm{nm}$ range. The spectrum of the $1 \% \mathrm{Pd} 3 \mathrm{O} 3$ doped thin film shows an emission onset near $450 \mathrm{~nm}$ with a primary emission peak at $477 \mathrm{~nm}$ and a second peak at $507 \mathrm{~nm}$. In contrast, $\mathrm{Pd} 3 \mathrm{O} 3$ in a dilute DCM solution contained a broad emission peak at $582 \mathrm{~nm}$ even after several dilutions and filtration. This low energy emission band is attributed to excimer emission which is supported by the excitation spectra which showed a shared origin for both the monomer and aggregate emission (Fig. S2 $\dagger$ ). Furthermore, a PMMA film cast from the same DCM solution shows no excimer emission, confirming the emission was not from ground state aggregates but rather excimer interactions (Fig. S3†). Doped thin films of $\mathrm{Pd} 3 \mathrm{O} 3$ in $26 \mathrm{mCPy}$ with doping concentrations of 5, 10, and 20 percent $\mathrm{Pd} 3 \mathrm{O} 3$ show the expected growth of the excimer emission with increasing concentration (Fig. S4†). The 


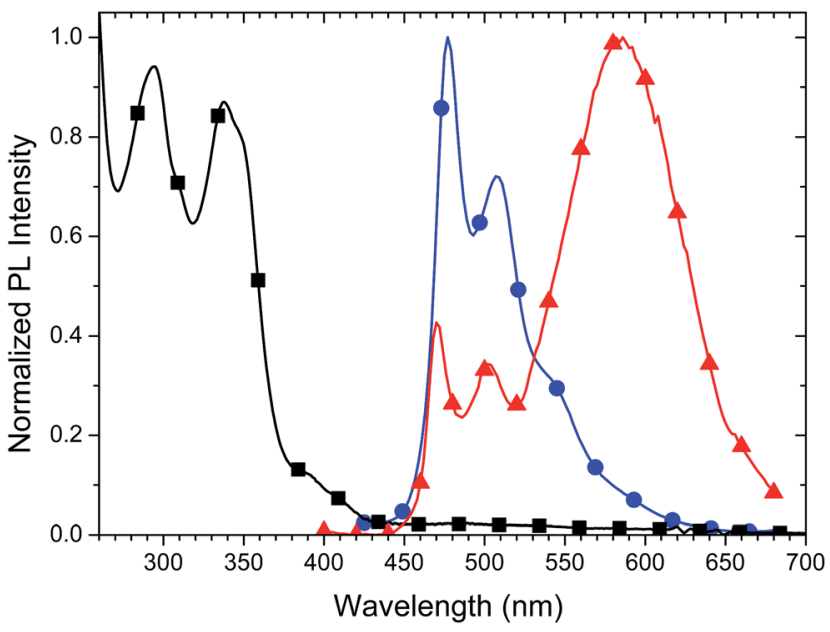

Fig. 2 The normalized absorption spectrum of $\mathrm{Pd} 3 \mathrm{O} 3$ in DCM (squares) and $\mathrm{PL}$ spectrum of a $1 \% \mathrm{Pd} 3 \mathrm{O} 3$ doped $26 \mathrm{mCPy}$ thin film (circles) and PL spectrum of a dilute DCM solution (triangles).

photoluminescent quantum yield (PLQY) of Pd3O3 in a doped PMMA film at room temperature was $87 \pm 10 \%$ and has a luminescent lifetime of $120 \mu \mathrm{s}$ indicating that while the radiative rate is low for Pd complexes such as these, the tetradentate complex structure greatly supresses the non-radiative rate, making this emitter a good candidate for efficient OLEDs. The oxidation and reduction potentials were $0.39 \mathrm{~V}$ and $-2.38 \mathrm{~V}$ respectively, determined by differential pulse voltammetry in dimethylformamide versus a ferrocene internal reference. These values correspond to a HOMO level of $5.15 \mathrm{eV}$ and a LUMO level of $1.95 \mathrm{eV}^{42,43}$ It is worth noting that these values fall within the redox gap of common host and transport materials such as mCBP or mCPy and is likely to improve charge and exciton confinement. ${ }^{32,42,43}$

\section{White OLED device performance}

To evaluate the performance of $\mathrm{Pd} 3 \mathrm{O} 3$ in a WOLED setting, devices were fabricated in a known efficient and charge confining structure: ITO/HATCN $(10 \mathrm{~nm}) / \mathrm{NPD}(40 \mathrm{~nm}) / \mathrm{TAPC}$ $(10 \mathrm{~nm}) / x \%$ Pd3O3:26mCPy $(25 \mathrm{~nm}) / \mathrm{DPPS}(10 \mathrm{~nm}) / \mathrm{BmPyPB}$ $(40 \mathrm{~nm}) / \mathrm{LiF} / \mathrm{Al}$ for dopant concentrations of $5 \%$ and $10 \%$ Pd3O3 by mass. ${ }^{4-46}$ HATCN is 1,4,5,8,9,11-hexaazatriphenylenehexacarbonitrile, NPD is $N, N^{\prime}$-diphenyl- $N, N^{\prime}$-bis(1-naphthyl)$1,1^{\prime}$-biphenyl-4,4"-diamine, TAPC is di-[4-( $N, N$-di-tolyl-amino)phenyl]cyclohexane, $26 \mathrm{mCPy}$ is 2,6-bis( $N$-carbazolyl)pyridine, DPPS is diphenyl-bis[4-(pyridin-3-yl)phenyl]silane, and BmPyPB is 1,3-bis[3,5-di(pyridin-3-yl)phenyl]benzene. As shown in Fig. 3, Pd3O3 devices at $5 \%$ and $10 \%$ dopant concentration show high peak EQEs of $23.9 \%$ and $24.2 \%$ respectively. This is the highest reported efficiencies for OLEDs employing Pd complexes regardless of emission color. ${ }^{33-35}$ The efficiency drops to $18.5 \%$ and $13.8 \%$ at $100 \mathrm{~cd} \mathrm{~m}^{-2}$ and $1000 \mathrm{~cd} \mathrm{~m}^{-2}$ for the $5 \%$ doped device and $19.3 \%$ and $14.3 \%$ at $100 \mathrm{~cd} \mathrm{~m}^{-2}$ and $1000 \mathrm{~cd} \mathrm{~m}^{-2}$ for the $10 \%$ doped device. This device efficiency roll-off is likely due to the combination of poor charge balance at high current density and the long PL emission lifetime of $120 \mu \mathrm{s.} .^{47}$ The peak

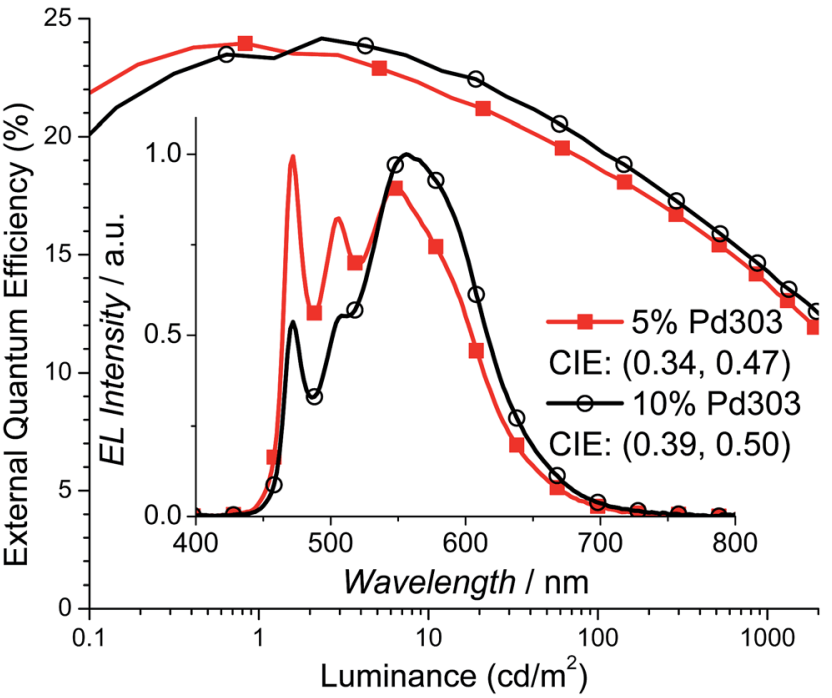

Fig. 3 External quantum efficiency versus luminance and electroluminescent spectra (inset) for $\mathrm{Pd} 3 \mathrm{O} 3$ devices with 5\% (squares) and 10\% (circles) dopant concentrations in the structure: ITO/HATCN/NPD/ TAPC/x\% Pd3O3:26mCPy/DPPS/BmPyPB/LiF/Al.

power efficiencies (PE) were also high for both these devices with $65.3 \mathrm{Lm}$ per W and $67.9 \mathrm{Lm}$ per W (Fig. S5 $\dagger$ ) for the $5 \%$ and $10 \%$ doped devices, albeit with non-ideal color coordinates of $(0.34,0.47)$ and $(0.39,0.50)$, respectively.

The emission spectra in the inset to Fig. 3 shows a monomer emission peak at $472 \mathrm{~nm}$ and a broad excimer peak at $550-600 \mathrm{~nm}$. At the dopant concentration of $5 \%$, the excimer peak and monomer peak are approximately equal heights yielding warm white light with CIE coordinates of $(0.34,0.47)$ and CRI of 53. When the concentration of $\mathrm{Pd} 3 \mathrm{O} 3$ is increased to $10 \%$, the excimer emission broadens and increases to approximately twice the height of the monomer emission. Consequently, the emission is orange with CIE coordinates of $(0.39,0.50)$ and a CRI of 52. It should also be noted that the monomer to excimer emission balance occurs at a much lower dopant concentration than many of the reported platinum complexes, yielding an emission spectrum with non-ideal CIE coordinates. ${ }^{38,39}$ This is due to the preferential stacking of $\mathrm{Pd} 3 \mathrm{O} 3$ molecules which was also reflected in the poor solubility of $\mathrm{Pd} 3 \mathrm{O} 3{ }^{48,49}$ Furthermore, the excimer emission drops off rapidly at $600 \mathrm{~nm}$ missing a significant portion of the red spectrum leading in part to the low CRI. Modifying the planar geometry nature of $\mathrm{Pd} 3 \mathrm{O} 3$ molecules by adding steric substitutional groups or using bulky bridging ligands will allow stronger molecular interaction between emissive materials and the host molecules and can tune the monomer and excimer emission colors to yield more ideal white color. ${ }^{50,51}$

\section{Devices with long operational lifetimes}

Currently, there are very few reports on the operational lifetime of white OLED devices and even less is known about the operational stability of excimer based WOLEDs. Due to the known instability of the TAPC and DPPS blocking materials, separate 
Pd3O3 devices were fabricated in stable device structure: ITO/HATCN (10 nm)/NPD (40 nm)/TrisPCz (10 nm)/EML/BAlq $(10 \mathrm{~nm}) / \mathrm{BPyTP}(40 \mathrm{~nm}) / \mathrm{LiF}(1 \mathrm{~nm}) / \mathrm{Al}$. The EML is varied as follows:

Device 1: 6\% Pd3O3: 26mCPy (30 nm).

Device 2: 10\% Pd3O3: 26mCPy (10 nm)/6\% Pd3O3: 26mCPy (20 nm).

Device 3: 20\% Pd3O3: 26mCPy (10 nm)/6\% Pd3O3: 26mCPy (20 nm).

Where TrisPCz is $9,9^{\prime}, 9^{\prime \prime}$-triphenyl-9H, $9^{\prime} \mathrm{H}, 9^{\prime \prime} \mathrm{H}-3,3^{\prime}: 6^{\prime} 3^{\prime \prime}$-tercarbazole, BAlq is bis(2-methyl-8-quinolinolato)(biphenyl-4-olato) aluminum, and BPyTP is 2,7-di(2,2'-bipyridin-5-yl)triphenylene, which are selected based on the previous literature reports related to stable fluorescent and phosphorescent devices. ${ }^{52,53}$ Devices 1 was fabricated with a fixed dopant concentration $6 \%$. As seen in Fig. 4a, the resulting spectrum for Device 1 contains a dominant excimer peak as well as a monomer peak at $472 \mathrm{~nm}$ that is approximately $50 \%$ of the excimer peak height. The monomer is suppressed compared to that of the $5 \%$ doped device in the charge confining structure despite their similar emissive layers. The reason for this reduction may be related to charge balance, morphological differences, or quenching of the monomeric emission by the TrisPCz blocking layers. Significant NPD emission is observed upon removal of the TrisPCz blocking layer in a similar device structure (Fig. S6†) suggesting significant exciton density near the EML/HTL interface. Despite this spectral change, the $6 \%$ doped device still achieved warm white emission with CIE coordinates of $(0.37,0.51)$ but a poor CRI of 48 . Device 1 achieved a peak EQE of $12.8 \%$ and a peak power efficiency of $33.1 \mathrm{Lm}$ per W, both of which dropped to $10.7 \%$ and $19.9 \mathrm{Lm}$ per W, respectively, at $1000 \mathrm{~cd} \mathrm{~m}^{-2}$. The device operational lifetime was measured at accelerated conditions of a constant current of $20 \mathrm{~mA} \mathrm{~cm}^{-2}$, which was summarized in Table 1. A nominal lifetime to $70 \%$ initial luminance $\left(\mathrm{LT}_{70}\right)$ for Device 1 was measured to be 40 hours at an initial luminance of $4927 \mathrm{~cd} \mathrm{~m}^{-2}$. This corresponds to roughly 600 hours at a practical luminance of $1000 \mathrm{~cd} \mathrm{~m}^{-2}$, using the formula $\operatorname{LT}\left(L_{1}\right)=\operatorname{LT}\left(L_{0}\right)\left(L_{0} / L_{1}\right)^{n}$, where $L_{1}$ is the desired luminance and the exponent $n$ is assumed to be a moderate value of 1.7.54

Previous reports have shown that while the use of TrisPCz improves device efficiency due to its charge blocking capabilities, the devices also suffers a loss in nominal operational lifetime. Such an effect is also observed for $\mathrm{Pd} 3 \mathrm{O} 3$ devices, and could be due to possible charge build-up at the interface of EML/EBL or due to inherent instabilities of the TrisPCz layer. ${ }^{47,52,53}$ In either case both high operational lifetimes and high efficiencies may be achieved by shifting the charge recombination zone toward the center of the emissive layer. Charge build-up near the TrisPCz interface can be explained by the energy level alignment of $\mathrm{Pd} 3 \mathrm{O} 3$ in the 26mCPy host. The LUMO level of Pd3O3 (1.95 eV) is a shallow trap in 26mCPy $(1.6 \mathrm{eV})$ whereas the HOMO level of $\mathrm{Pd} 3 \mathrm{O} 3(5.15 \mathrm{eV})$ is a much deeper trap in $26 \mathrm{mCPy}(6.0 \mathrm{eV})$. This would likely lead to an imbalance in charge transport due to the higher energetic barriers for the successive hopping of holes in and out of the traps. To compensate for the imbalance the doping concentration near the electron blocking layer was increased to $10 \%$ for Device 2 and $20 \%$ for Device 3. Increasing the doping concentration expectedly increased the excimer contribution to the emission ultimately leading to a shift in color from white to orange with CIE coordinates of $(0.48,0.50)$ for Device 3 . Both the
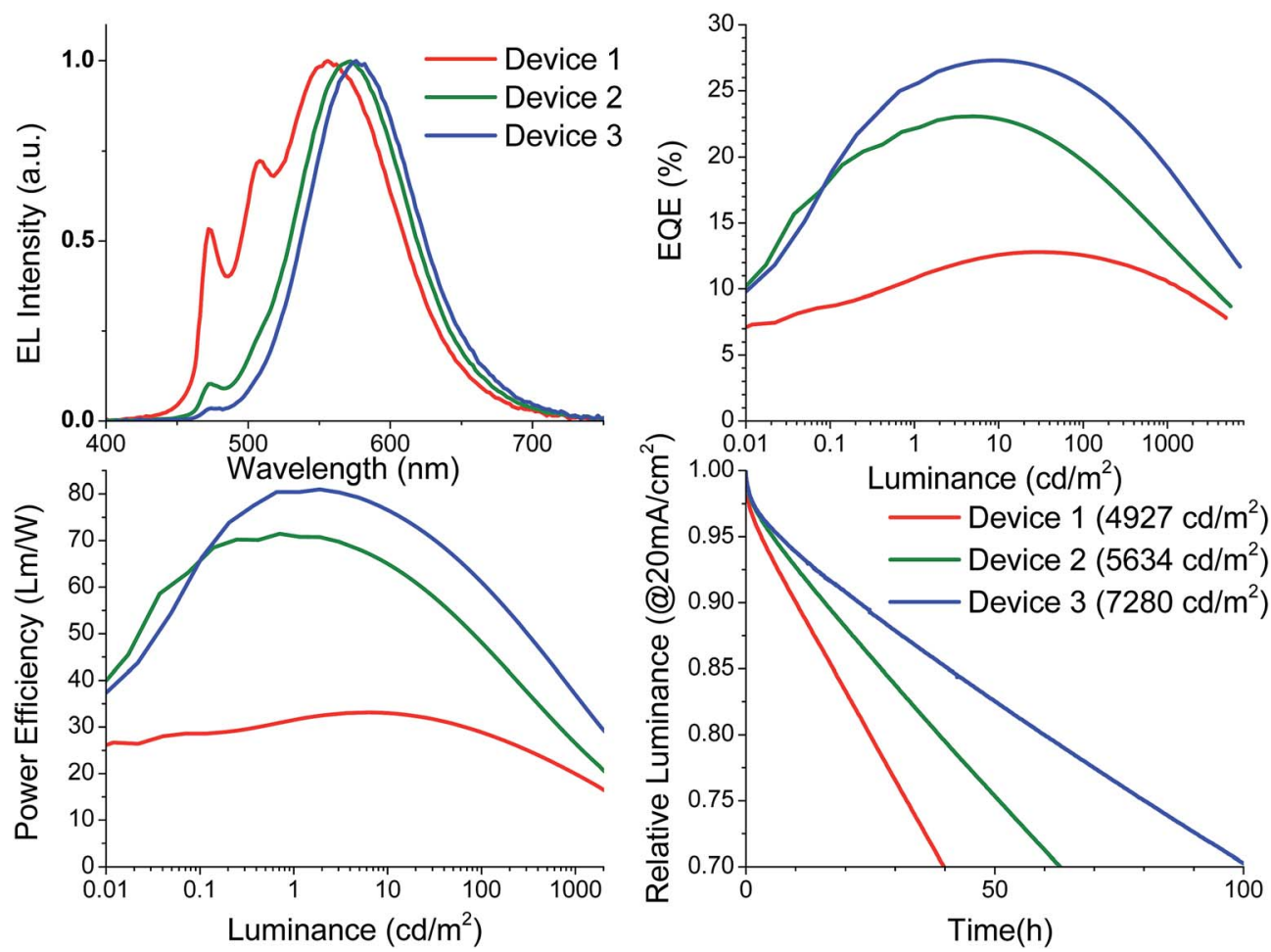

Fig. 4 (a) Electroluminescent spectra, (b)external quantum efficiency versus luminance, (c) power efficiency versus luminance and (d) operational lifetime for Pd3O3 in Device 1 (red), Device 2 (green), and Device 3 (blue). The device operational lifetime was measured at a constant drive current of $20 \mathrm{~mA} \mathrm{~cm}^{-2}$. 
Table 1 Summary of device performance for stable devices of Pd3O3

\begin{tabular}{|c|c|c|c|c|c|c|c|c|c|}
\hline Device & CRI & CIE & \multicolumn{2}{|c|}{ EQE (\%) } & \multicolumn{2}{|c|}{ PE (Lm per W) } & \multicolumn{3}{|l|}{$\mathrm{LT}_{70}$} \\
\hline 2 & 45 & $(0.45,0.51)$ & 23.1 & 13.6 & 71.4 & 26.5 & 5634 & 63 & 1190 \\
\hline 3 & 43 & $(0.48,0.50)$ & 27.3 & 19.2 & 81.0 & 36.9 & 7280 & 101 & 2950 \\
\hline
\end{tabular}

EQE and power efficiencies of the devices were improved dramatically yielding a peak EQE of $27.3 \%$ and a power efficiency of $81 \mathrm{Lm}$ per $\mathrm{W}$ for Device 3. Remarkably, at $1000 \mathrm{~cd} \mathrm{~m}^{-2}$ the EQE remained at $19.2 \%$ and the power efficiency at $36.9 \mathrm{Lm}$ per $\mathrm{W}$ despite the long phosphorescent lifetime of $120 \mu \mathrm{s}$. Accelerated operational lifetime testing was carried out on Device 2 and 3, exhibiting improved nominal operational lifetimes at $20 \mathrm{~mA} \mathrm{~cm}^{-2}$ compared to Device 1 . The use of a $10 \%$ doped layer for Device 2 achieved an $\mathrm{LT}_{70}$ of 62 hours at $5634 \mathrm{~cd} \mathrm{~m}^{-2}$ corresponding to 1190 hours at $1000 \mathrm{~cd} \mathrm{~m}^{-2}$, while the use of a $20 \%$ doped layer further improved the operational lifetime to an $\mathrm{LT}_{70}$ of 101 hours at $7280 \mathrm{~cd} \mathrm{~m}^{-2}$ corresponding to 2950 hours at $1000 \mathrm{~cd} \mathrm{~m}^{-2}$. The higher efficiencies, low roll-off, and extended operational lifetimes all suggest a significant improvement in charge balance inside the device. ${ }^{47}$ The ability to simultaneously achieve long lifetime and high efficiency in the same device represents a major step forward in the development of stable single doped white OLEDs. The nearly $20 \% \mathrm{EQE}$ and $\mathrm{LT}_{70}$ of 3000 hours at $1000 \mathrm{~cd} \mathrm{~m}^{-2}$ push both palladium complexes and their use in excimer based white OLEDs to the forefront of academic efforts in OLED development. Furthermore, with incorporation of light outcoupling techniques, doubling the luminance at a given driving condition could be reasonably expected to yield lifetimes of 10000 hours at $1000 \mathrm{~cd} \mathrm{~m}^{-2}$ which approaches the minimum commercialization requirement. ${ }^{54}$ Our progress represents a substantial improvement upon previous reports of Pt-based excimers which demonstrated less than $100 \mathrm{~h}$ for Pt7O7 or $400 \mathrm{~h}$ for Pt $1 \mathrm{O} 2 \mathrm{me}_{2}$ at $1000 \mathrm{~cd} \mathrm{~m}^{-2}$, both of which were only in the range of $8-12 \%$ efficient. ${ }^{38,39}$ This indicates the importance of maximizing emissive material performance through judicious molecular design and rational device architecture optimization.

\section{Conclusions}

In summary, an efficient and stable blue emitting palladium complex, $\mathrm{Pd} 3 \mathrm{O} 3$, was designed and prepared, enabling efficient and stable single-doped WOLED for lighting applications. Devices employing this complex achieved peak efficiencies over $27 \%$ which are comparable to the state of the art Ir and Pt analogs in a similar device structure, demonstrating the value of this new class of complexes. ${ }^{10-25}$ Furthermore, the ability to achieve higher energy emission for a given cyclometalating ligand makes this class of complex particular interesting for both blue and white OLED applications. One stable excimer emitting device achieved a peak efficiency $27.3 \%$ and $81 \mathrm{Lm}$ per $\mathrm{W}$ while also having a power efficiency of $36.9 \mathrm{Lm}$ per $\mathrm{W}$ and operational lifetime of nearly $3000 \mathrm{~h}$ at $1000 \mathrm{~cd} \mathrm{~m}^{-2}$. Further development of the emissive materials and fine tuning of the emissive layer is expected to improve the color quality to achieve efficient and stable single doped white OLEDs. Thus, the introduction of efficient and stable palladium complexes and their use in single doped white OLEDs could provide a route for efficient low-cost solid state lighting. While this particular complex rapidly formed excimers even at low doping concentrations, this material and corresponding device design demonstrates the potential for simultaneously achieving high efficiencies and long operational lifetimes in devices with high triplet energy emitters. It is worth noting that $\mathrm{Pd} 3 \mathrm{O} 3$ demonstrates the longest device operational lifetime in the academic literature for any phosphorescent emitter with triplet energy of over $2.6 \mathrm{eV}^{29,32}$ The knowledge and experience gained from these results will open a new avenue of developing stable and efficient blue phosphorescent emitters for display and lighting applications.

\section{Experimental}

\section{General synthetic procedure}

All commercial reagents were purchased and used as received without further purification. $\mathrm{Pd}(\mathrm{OAc})_{2}$ was purchased from Pressure Chemical Co. $n$ - $\mathrm{Bu}_{4} \mathrm{NBr}$, CuI, 2-(tributylstannyl)pyridine and 2-picolinic acid were purchased from Sigma Aldrich. Silica gel $(40-60 \mu \mathrm{m})$ was purchased from Agela Technologies and BDH. Solvents DMSO, toluene (low water), acetate acid were purchased from Alfa Aesar, J. T. Baker, Fluke and BDH respectively. All reactions were carried out under an inert $\mathrm{N}_{2}$ atmosphere in oven-dried glassware. External bath temperatures were used to record all reaction temperatures. Flash column chromatography was carried out with silica gel. Proton and carbon NMR spectra ( ${ }^{1} \mathrm{H}$ NMR and ${ }^{13} \mathrm{C}$ NMR) were recorded in dimethyl sulfoxide- $d_{6}$ (DMSO- $d_{6}$ ) on a Varian $400 \mathrm{MHz}$ NMR spectrometer. The solvent residual peak (DMSO- $d_{6}$ ) was calibrated to $2.50 \mathrm{ppm}$ for ${ }^{1} \mathrm{H}$ NMR and $39.52 \mathrm{ppm}$ for ${ }^{13} \mathrm{C}$ NMR. Multiplicities are abbreviated as follows: $\mathrm{s}=$ singlet, $\mathrm{d}=$ doublet, $\mathrm{t}=$ triplet, $\mathrm{br}=$ broad, $\mathrm{m}=$ multiplet.

\section{The synthesis of $\mathrm{Pd} 3 \mathrm{O} 3$}

2-(3-(3-(Pyridin-2-yl)phenoxy)phenyl)pyridine ( $470 \mathrm{mg}, 1.45 \mathrm{mmol}$ ), $\mathrm{Pd}(\mathrm{OAc})_{2}(348 \mathrm{mg}, 1.55 \mathrm{mmol})$, and $n-\mathrm{Bu}_{4} \mathrm{NBr}(48 \mathrm{mg}, 0.149 \mathrm{mmol})$ were added into a $100 \mathrm{ml}$ three-neck round-bottom flask, then $30 \mathrm{ml}$ acetic acid was added. The mixture was bubbled with nitrogen for 30 minutes then stirred at ambient temperature for 
12 hours. The mixture was heated in an oil bath at a temperature of $110^{\circ} \mathrm{C}$ for another 72 hours. $100 \mathrm{ml}$ of water was added after the mixture was cooled down to room temperature. The precipitate was collected through filtration, washed with water for three times then dried in air. The collected solid was purified through column chromatography on silica gel using dichloromethane as eluent to afford the desired palladium complex $\mathrm{Pd} 3 \mathrm{O} 3$ as a light yellow solid $390 \mathrm{mg}$ in $63 \%$ yield. ${ }^{1} \mathrm{H}$ NMR (DMSO- $d_{6}, 400 \mathrm{MHz}$ ): $\delta 7.16(\mathrm{~d}, J=$ $7.6 \mathrm{~Hz}, 2 \mathrm{H}), 7.27$ (t, $J=8.0 \mathrm{~Hz}, 2 \mathrm{H}), 7.55$ (t, $J=6.4 \mathrm{~Hz}, 2 \mathrm{H}), 7.74$ $(\mathrm{d}, J=7.2 \mathrm{~Hz} \mathrm{2H}), 8.09-8.15(\mathrm{~m}, 2 \mathrm{H}), 8.28(\mathrm{~d}, J=8.4 \mathrm{~Hz}, 2 \mathrm{H}), 8.96$ (d, $J=5.2 \mathrm{~Hz}, 2 \mathrm{H}$ ). MS (APCI $\left.{ }^{+}\right) m / z:[\mathrm{M}]^{+}$calcd for $\mathrm{C}_{22} \mathrm{H}_{15} 0 \mathrm{~N}_{2} \mathrm{OPd}$ 429.0219, found 429.0232. Anal. calcd for $\mathrm{C}_{22} \mathrm{H}_{14} \mathrm{~N}_{2} \mathrm{OPd}$ : C, 61.63; $\mathrm{H}, 3.29$; N, 6.53; found: C, 61.70; H, 3.31; N, 6.62.

\section{Materials}

TAPC (di-[4-( $N, N$-di-tolyl-amino)-phenyl]cyclohexane) ${ }^{46}$ TrisPCz $\left(9,9^{\prime}, 9^{\prime \prime}\right.$-triphenyl-9H, $9^{\prime} \mathrm{H}, 9^{\prime \prime} \mathrm{H}-3,3^{\prime}: 6^{\prime} 3^{\prime \prime}$-tercarbazole) ${ }^{53} \quad 26 \mathrm{mCPy}$ (2,6-bis( $N$-carbazolyl)pyridine), ${ }^{49}$ DPPS (diphenyl-bis[4-(pyridin3-yl)phenyl]silane), ${ }^{\mathbf{4 4}} \quad$ BmPyPB $\quad$ (1,3-bis[3,5-di(pyridin-3-yl) phenyl]benzene), ${ }^{45}$ and BPyTP (2,7-di(2,2'-bipyridin-5-yl)triphenylene $)^{53}$ were all synthesized following previous literature reports. HATCN (1,4,5,8,9,11-hexaazatriphenylene-hexacarbonitrile), NPD ( $N, N^{\prime}$-diphenyl- $N, N^{\prime}$-bis(1-naphthyl)-1, $1^{\prime}$-biphenyl-4, $4^{\prime \prime}$-diamine), BAlq (bis(2-methyl-8-quinolinolato)(biphenyl-4-olato) aluminum), and mCBP (3,3-di(9H-carbazol-9-yl) biphenyl) were all provided from commercial suppliers. All materials were sublimed in a 4-zone thermal gradient furnace at pressures of $10^{-5}$ torr prior to use.

\section{Materials characterization}

The photoluminescent spectra were measured on a Horiba Jobin Yvon FluoroLog-3 spectrometer. The absorption spectrum was measured on an Agilent 8453 UV-visible spectrometer. Doped thin films for photoluminescent quantum yield (PLQY) measurements were fabricated by the drop-cast process of Pd3O3 in a PMMA host. The PLQY measurements were carried out on a Hamamatsu absolute PL quantum yield spectrometer model C11347. It should be noted that the error bar may be large for the PLQY measurement with emissive materials which have long lifetimes ( $>100 \mu \mathrm{s})$ due to quenching by residual oxygen in the integration sphere. Cyclic voltammetry and differential pulsed voltammetry were performed on a CHI610B electrochemical analyzer in a solution of anhydrous DMF using $0.1 \mathrm{M}$ tetra(nbutyl) ammonium hexafluorophosphate as the supporting electrolyte. A ferrocene/ferrocenium $\left(\mathrm{Fc} / \mathrm{Fc}^{+}\right)$redox couple used as an internal reference. The HOMO and LUMO values were determined following literature reported fits relating the electrochemical potentials to the values determined via ultraviolet photoemission spectroscopy and inverse photoelectron spectroscopy, respectively. ${ }^{\mathbf{4 2 , 4 3}}$ Mass spectra were recorded on JEOL GCmate gas chromatograph/mass spectrometer. Elemental analysis was carried out with a PerkinElmer 2400 CHN Elemental Analyzer.

\section{Device fabrication and characterization}

Devices were fabricated on pre-patterned substrates of ITO on glass purchased from Optics Balzers (50 $\Omega$ per sq.). Prior to deposition substrates were cleaned by a gentle scrub followed by subsequent sonication in water, acetone, and isopropanol. Organic layers were deposited by vacuum thermal evaporation in a custom made chamber by Travato Man. Inc. Base pressures were kept between $10^{-8}$ to $10^{-7}$ torr and deposition rates were kept between $0.5-1.0 \AA \mathrm{s}^{-1}$. A $1 \mathrm{~nm} \mathrm{LiF}$ buffer layer was deposited at $0.2 \AA \mathrm{s}^{-1}$. Al cathodes were deposited without breaking vacuum at $1-2 \AA \mathrm{s}^{-1}$ through a shadow mask defining a device area of $4 \mathrm{~mm}^{2}$.

High efficiency devices were fabricated in the structure: ITO/ HATCN $(10 \mathrm{~nm}) / \mathrm{NPD}(40 \mathrm{~nm}) / \mathrm{TAPC}(10 \mathrm{~nm}) / x \%$ Pd3O3:26mCPy $(25 \mathrm{~nm}) / \mathrm{DPPS}(10 \mathrm{~nm}) / \mathrm{BmPyPB}(40 \mathrm{~nm}) / \mathrm{LiF} / \mathrm{Al}$ where $x=5 \%$ or $10 \%$. For stable devices the following structure was used: ITO/ HATCN $\quad(10 \quad \mathrm{~nm}) / \mathrm{NPD} \quad(40 \quad \mathrm{~nm}) / \mathrm{TrisPCz} \quad(10 \mathrm{~nm}) / \mathrm{EML} / \mathrm{BAlq}$ $(10 \mathrm{~nm}) / \mathrm{BPyTP}(40 \mathrm{~nm}) / \mathrm{LiF}(1 \mathrm{~nm}) / \mathrm{Al}$. The EML is varied as follows:

Device 1: 6\% Pd3O3: 26mCPy (30 nm).

Device 2: 10\% Pd3O3: 26mCPy (10 nm)/6\% Pd3O3: 26mCPy (20 $\mathrm{nm})$.

Device 3: 20\% Pd3O3: 26mCPy (10 nm)/6\% Pd3O3: 26mCPy (20 nm).

Current-voltage-luminance characteristics were taken with a Keithley 2400 Source-Meter and a Newport 818 Si photodiode inside a nitrogen-filled glove-box with all devices assumed to be Lambertian emitters. Accelerated lifetime testing was performed in a nitrogen filled glove box without encapsulation at a constant current of $20 \mathrm{~mA} \mathrm{~cm}{ }^{-2}$. Extrapolated lifetimes were approximated using the formula $\operatorname{LT}\left(L_{1}\right)=\operatorname{LT}\left(L_{0}\right)\left(L_{0} / L_{1}\right)^{1.7} \cdot{ }^{54} \mathrm{EL}$ spectra were taken at $1 \mathrm{~mA} \mathrm{~cm}{ }^{-2}$ using a calibrated ocean optics USB 2000 spectrometer.

\section{Conflicts of interest}

There are no conflicts of interest to declare.

\section{Acknowledgements}

The authors thank Dr Jason Brooks from Universal Display Corporation for performing the luminescent lifetime and PLQY measurement of $\mathrm{Pd} 3 \mathrm{O} 3$ samples. The authors thank Universal Display Corporation, the National Science Foundation (CHE0748867), Department of Energy (contract no. EE0007090) and Advanced Photovoltaics Center for partial support of this work.

\section{References}

1 M. C. Gather, A. Köhnen and K. Meerholz, White Organic Light-Emitting Diodes, Adv. Mater., 2011, 23, 233-248.

2 B. W. D'Andrade and S. R. Forrest, White Organic LightEmitting Devices for Solid-State Lighting, Adv. Mater., 2004, 16, 1585-1595.

3 F. So, J. Kido and P. Burrows, Organic Light-Emitting Devices for Solid-State Lighting, MRS Bull., 2008, 33, 663-669.

4 M. A. Baldo, et al., Highly efficient phosphorescent emission from organic electroluminescent devices, Nature, 1998, 395, 151-154.

5 M. A. Baldo, S. Lamansky, P. E. Burrows, M. E. Thompson and S. R. Forrest, Very high-efficiency green organic light- 
emitting devices based on electrophosphorescence, Appl. Phys. Lett., 1999, 75, 4-6.

6 C. Adachi, M. A. Baldo, M. E. Thompson and S. R. Forrest, Nearly $100 \%$ internal phosphorescence efficiency in an organic light-emitting device, J. Appl. Phys., 2001, 90, 5048-5051.

7 B. W. D'Andrade, R. J. Holmes and S. R. Forrest, Efficient Organic Electrophosphorescent White-Light-Emitting Device with a Triple Doped Emissive Layer, Adv. Mater., 2004, 16, 624-628.

8 S. Reineke, et al., White organic light-emitting diodes with fluorescent tube efficiency, Nature, 2009, 459, 234-238.

9 K. T. Kamtekar, A. P. Monkman and M. R. Bryce, Recent Advances in White Organic Light-Emitting Materials and Devices (WOLEDs), Adv. Mater., 2010, 22, 572-582.

10 Y. Sun and S. R. Forrest, High-efficiency white organic light emitting devices with three separate phosphorescent emission layers, Appl. Phys. Lett., 2007, 91, 263503.

$11 \mathrm{~J}$. Ye, et al., Management of Singlet and Triplet Excitons in a Single Emission Layer: A Simple Approach for a HighEfficiency Fluorescence/Phosphorescence Hybrid White Organic Light-Emitting Device, Adv. Mater., 2012, 24, 34103414.

12 Q.-D. Ou, et al., Extremely Efficient White Organic LightEmitting Diodes for General Lighting, Adv. Funct. Mater., 2014, 24, 7249-7256.

13 T. Higuchi, H. Nakanotani and C. Adachi, High-Efficiency White Organic Light-Emitting Diodes Based on a Blue Thermally Activated Delayed Fluorescent Emitter Combined with Green and Red Fluorescent Emitters, Adv. Mater., 2015, 27, 2019-2023.

14 B. W. D'Andrade, J. Brooks, V. Adamovich, M. E. Thompson and S. R. Forrest, White Light Emission Using Triplet Excimers in Electrophosphorescent Organic Light-Emitting Devices, Adv. Mater., 2002, 14, 1032-1036.

15 X. Yang, Z. Wang, S. Madakuni, J. Li and G. E. Jabbour, Efficient Blue- and White-Emitting Electrophosphorescent Devices Based on Platinum(II) [1,3-Difluoro-4,6-di(2pyridinyl)benzene] Chloride, Adv. Mater., 2008, 20, 24052409.

16 S. C. F. Kui, et al., Robust Phosphorescent Platinum(II) Complexes Containing Tetradentate $\mathrm{O}^{\wedge} \mathrm{N}^{\wedge} \mathrm{C}^{\wedge} \mathrm{N}$ Ligands: Excimeric Excited State and Application in Organic WhiteLight-Emitting Diodes, Chem.-Eur. J., 2013, 19, 69-73.

17 L. Murphy, P. Brulatti, V. Fattori, M. Cocchi and J. A. G. Williams, Blue-shifting the monomer and excimer phosphorescence of tridentate cyclometalated platinum(II) complexes for optimal white-light OLEDs, Chem. Commun., 2012, 48, 5817-5819.

18 T. Fleetham, J. Ecton, Z. Wang, N. Bakken and J. Li, SingleDoped White Organic Light-Emitting Device with an External Quantum Efficiency Over 20\%, Adv. Mater., 2013, 25, 2573-2576.

19 C. H. Hsieh, et al., Design and Synthesis of Iridium Bis(carbene) Complexes for Efficient Blue Electrophosphorescence, Chem.Eur. J., 2011, 17, 9180-9187.

20 S. Haneder, et al., Controlling the Radiative Rate of DeepBlue Electrophosphorescent Organometallic Complexes by
Singlet-Triplet Gap Engineering, Adv. Mater., 2008, 20, 3325-3330.

21 C.-F. Chang, et al., Highly Efficient Blue-Emitting Iridium(III) Carbene Complexes and Phosphorescent OLEDs, Angew. Chem., Int. Ed., 2008, 47, 4542-4545.

22 T. Fleetham, G. Li, L. Wen and J. Li, Efficient "Pure" Blue OLEDs Employing Tetradentate Pt Complexes with a Narrow Spectral Bandwidth, Adv. Mater., 2014, 2, 71167121.

23 S. O. Jeon, S. E. Jang, H. S. Son and J. Y. Lee, External Quantum Efficiency Above 20\% in Deep Blue Phosphorescent Organic Light-Emitting Diodes, Adv. Mater., 2011, 23, 1436-1441.

$24 \mathrm{H}$. Sasabe, et al., High-Efficiency Blue and White Organic Light-Emitting Devices Incorporating a Blue Iridium Carbene Complex, Adv. Mater., 2010, 22, 5003-5007.

25 T. Fleetham, Z. Wang and J. Li, Efficient deep blue electrophosphorescent devices based on platinum(II) bis(nmethyl-imidazolyl)benzene chloride, Org. Electron., 2012, 13, 1430-1435.

26 X. Hang, T. Fleetham, E. Turner, J. Brooks and J. Li, Highly Efficient Blue-Emitting Cyclometalated Platinum(II) Complexes by Judicious Molecular Design, Angew. Chem., Int. Ed., 2013, 52, 6753-6756.

27 K. S. Yook and J. Y. Lee, Organic Materials for Deep Blue Phosphorescent Organic Light-Emitting Diodes, Adv. Mater., 2012, 24, 3169-3190.

28 K. P. Klubek, C. W. Tang and L. J. Rothberg, Investigation of blue phosphorescent organic light-emitting diode host and dopant stability, Org. Electron., 2014, 15, 1312-1316.

29 Y. Zhang, J. Lee and S. R. Forrest, Tenfold increase in the lifetime of blue phosphorescent organic light-emitting diodes, Nat. Commun., 2014, 5, 5008.

30 R. Seifert, et al., Chemical degradation mechanisms of highly efficient blue phosphorescent emitters used for organic light emitting diodes, Org. Electron., 2013, 14, 115123.

$31 \mathrm{~V}$. Sivasubramaniam, et al., Fluorine cleavage of the light blue heteroleptic triplet emitter FIrpic, J. Fluorine Chem., 2009, 130, 640-649.

32 T. B. Fleetham, L. Huang, K. Klimes, J. Brooks and J. Li, Tetradentate Pt(II) Complexes with 6-Membered Chelate Rings: A New Route for Stable and Efficient Blue Organic Light Emitting Diodes, Chem. Mater., 2016, 28, 3276-3282.

33 P. K. Chow, et al., Strongly Phosphorescent Palladium(II) Complexes of Tetradentate Ligands with Mixed Oxygen, Carbon, and Nitrogen Donor Atoms: Photophysics, Photochemistry, and Applications, Angew. Chem., Int. Ed., 2013, 52, 11775-11779.

$34 \mathrm{~K}$. Li, et al., Light-emitting platinum(II) complexes supported by tetradentate dianionic bis(N-heterocyclic carbene) ligands: towards robust blue electrophosphors, Chem. Sci., 2013, 4, 2630-2644.

35 Z.-Q. Zhu, T. Fleetham, E. Turner and J. Li, Harvesting All Electrogenerated Excitons Through Metal Assisted Delayed Fluorescent Materials, Adv. Mater., 2015, 27, 2533-2537.

36 S.-W. Lai, et al., Luminescent Mononuclear and Binuclear Cyclometalated Palladium(II) Complexes of 6-Phenyl-2,2'- 
bipyridines: Spectroscopic and Structural Comparisons with Platinum(II) Analogues, Inorg. Chem., 2000, 39, 255-262.

37 S. Roy, S. Pramanik, T. Ghorui and K. Pramanik, Insight into luminescent bisazoaromatic CNN pincer palladacycle: synthesis, structure, electrochemistry and some catalytic applications in C-C coupling, RSC Adv., 2015, 5, 22544-22559.

38 G. Li, T. Fleetham and J. Li, Efficient and Stable White Organic Light-Emitting Diodes Employing a Single Emitter, Adv. Mater., 2104, 26, 2931-2936.

39 T. Fleetham, L. Haung and J. Li, Tetradentate Platinum Complexes for Efficient and Stable Excimer-Based White OLEDs, Adv. Funct. Mater., 2014, 24, 6066-6073.

40 E. Turner, N. Bakken and J. Li, Cyclometalated Platinum Complexes with Luminescent Quantum Yields Approaching 100\%, Inorg. Chem., 2013, 52, 7344-7351.

41 W. J. Finkenzeller and H. Yersin, Temperature dependence, decay dynamics, and magnetic field properties, Chem. Phys. Lett., 2003, 377, 299-305.

42 B. W. D'Andrade, et al., Relationship between the ionization and oxidation potentials of molecular organic semiconductors, Org. Electron., 2005, 6, 11-20.

43 P. Djurovich, E. I. Mayo, S. R. Forrest and M. E. Thompson, Measurement of the lowest unoccupied molecular orbital energies of molecular organic semiconductors, Org. Electron., 2009, 10, 515-520.

44 L. Xiao, S.-J. Su, Y. Agata, H. Lan and J. Kido, Nearly 100\% Internal Quantum Efficiency in an Organic Blue-Light Electrophosphorescent Device Using a Weak Electron Transporting Material with a Wide Energy Gap, $A d v$. Mater., 2009, 21, 1271-1274.

45 H. Sasabe, et al., Wide-Energy-Gap Electron-Transport Materials Containing 3,5-Dipyridylphenyl Moieties for an
Ultra High Efficiency Blue Organic Light-Emitting Device, Chem. Mater., 2008, 20, 5951-5953.

46 N. Chopra, et al., High efficiency and low roll-off blue phosphorescent organic light-emitting devices using mixed host architecture, Appl. Phys. Lett., 2010, 97, 033304.

47 C. Murawski, K. Leo and M. C. Gather, Efficiency roll-off in organic light-emitting diodes, Adv. Mater., 2013, 25, 6801-6827.

$48 \mathrm{~V}$. Adamovich, et al., High efficiency single dopant white electrophosphorescent light emitting diodes, New J. Chem., 2002, 26, 1171-1178.

49 E. L. Williams, K. Haavisto, J. Li and G. E. Jabbour, ExcimerBased White Phosphorescent Organic Light-Emitting Diodes with Nearly $100 \%$ Internal Quantum Efficiency, Adv. Mater., 2007, 19, 197-202.

50 B. Ma, et al., Synthetic Control of Pt...Pt Separation and Photophysics of Binuclear Platinum Complexes, J. Am. Chem. Soc., 2005, 127, 28-29.

51 S. J. Farley, D. L. Rochester, A. L. Thompson, J. A. K. Howard and J. A. G. Williams, Controlling Emission Energy, SelfQuenching, and Excimer Formation in Highly Luminescent $\mathrm{N} \wedge \mathrm{C} \wedge \mathrm{N}$-Coordinated Platinum(II) Complexes, Inorg. Chem., 2005, 44, 9690-9703.

52 G. Li, T. Fleetham, E. Turner, X.-C. Hang and J. Li, Highly Efficient and Stable Narrow-Band Phosphorescent Emitters for OLED Applications, Adv. Opt. Mater., 2015, 3, 390-397.

53 H. Nakanotani, K. Masui, J. Nishide, T. Shibata and C. Adachi, Promising operational stability of high-efficiency organic light-emitting diodes based on thermally activated delayed fluorescence, Sci. Rep., 2013, 3, 2127.

54 C. Féry, B. Racine, D. Vaufrey, H. Doyeux and S. Cinà, Physical mechanism responsible for the stretched exponential decay behavior of aging organic light-emitting diodes, Appl. Phys. Lett., 2005, 87, 213502. 